\title{
Shear Wave Induced Resonance Elastography of Venous Thrombi: A Proof-of-Concept
}

\author{
Cédric Schmitt, Emmanuel Montagnon, Anis Hadj Henni, Shijie Qi, and Guy Cloutier*
}

\begin{abstract}
Shear wave induced resonance elastography (SWIRE) is proposed for deep venous thrombosis (DVT) elasticity assessment. This new imaging technique takes advantage of properly polarized shear waves to induce resonance of a confined mechanical heterogeneity. Realistic phantoms $(n=9)$ of DVT total and partial clot occlusions with elasticities from 406 to $3561 \mathrm{~Pa}$ were built for in vitro experiments. An ex vivo study was also performed to evaluate the elasticity of two fresh porcine venous thrombi in a pig model. Transient shear waves at $45-205 \mathrm{~Hz}$ were generated by the vibration of a rigid plate (plane wavefront) or by a needle to simulate a radiation pressure on a line segment (cylindrical wavefront). Induced propagation of shear waves was imaged with an ultrafast ultrasound scanner and a finite element method was developed to simulate tested experimental conditions. An inverse problem was then formulated considering the first resonance frequency of the DVT inclusion. Elasticity agreements between SWIRE and a reference spectroscopy instrument (RheoSpectris) were found in vitro for total clots either in plane $\left(\mathrm{r}^{2}=0.989\right)$ or cylindrical $\left(r^{2}=0.986\right)$ wavefront configurations. For total and partial clots, elasticity estimation errors were $9.0 \pm 4.6 \%$ and $9.3 \pm 11.3 \%$, respectively. Ex vivo, the blood clot elasticity was $498 \pm 58 \mathrm{~Pa}$ within the inferior vena cava and $436 \pm 45 \mathrm{~Pa}$ in the right common iliac vein $(p=0.22)$. To conclude, the SWIRE technique seems feasible to quantitatively assess blood clot elasticity in the context of DVT ultrasound imaging.
\end{abstract}

Index Terms-Deep venous thrombosis (DVT), dynamic elastography, mechanical resonance, ultrasound imaging.

\section{INTRODUCTION}

$\mathbf{S}$ TATISTICS on venous thromboembolism (VTE) reveal an incidence of 100 per 100000 persons each year in the United States [1]. About $66 \%$ of diagnosed VTE occur in lower

Manuscript received August 21,2012; revised November 19, 2012; accepted November 25, 2012. Date of publication December 11, 2012; date of current version February 27, 2013. This work was supported by the Canadian Institutes of Health Research under Grant MOP-84358. Asterisk indicates corresponding author.

C. Schmitt and E. Montagnon are with the Laboratory of Biorheology and Medical Ultrasonics, University of Montreal Hospital Research Center (CRCHUM), Montréal, QC, H2L 2W5 Canada, and also with the Institute of Biomedical Engineering, University of Montreal, Montréal, QC, H3C 3J7 Canada (e-mail: aioli51@gmail.com; manumontagnon@yahoo.fr).

A. Hadj Henni is with the Laboratory of Biorheology and Medical Ultrasonics, University of Montreal Hospital Research Center (CRCHUM), Montréal, QC, H2L 2W5 Canada (e-mail: anis.hadjhenni@gmail.com).

S. Qi is with the Laboratory of Experimental Microsurgery, University of Montreal Hospital Research Center (CRCHUM), and Department of Surgery, University of Montreal, Montréal, QC, H3T 1J4 Canada (e-mail: shijie.qi@umontreal.ca).

${ }^{*} \mathrm{G}$. Cloutier is with the Laboratory of Biorheology and Medical Ultrasonics, University of Montreal Hospital Research Center (CRCHUM), Montréal, QC, H2L 2W5 Canada, and also with the Institute of Biomedical Engineering, University of Montreal, Montréal, QC, H3C 3J7 Canada, and also with the Department of Radiology, Radio-Oncology and Nuclear Medicine, University of Montreal, Montréal, QC, H3T 1J4 Canada (e-mail: guy.cloutier@umontreal.ca).

Color versions of one or more of the figures in this paper are available online at http://ieeexplore.ieee.org.

Digital Object Identifier 10.1109/TMI.2012.2231093 limbs where a deep vein thrombus (DVT), usually above the knee, migrates to trigger a pulmonary embolism (PE). Mortality of DVT cases is $6 \%$ within one month of diagnosis. Patients experiencing a first DVT are diagnosed by clinical symptoms and blood tests (D-dimer) combined with venous ultrasonography (i.e., compression ultrasonography and color Doppler) [2]. Thirty percent of DVT patients will develop recurrent episodes within eight years, with an associated mortality of $2 \%$ [3]. The therapeutic strategy greatly depends on the nature of the thrombus and on its severity (total or partial occlusion). If it is a new episode of DVT, namely an acute DVT, the optimal treatment consists of heparin or low-molecular weight heparin intravenously injected followed by oral anticoagulants [4]. In contrast, for a chronic thrombus older than six months, the therapy is different and consists of oral anticoagulants alone because heparin can induce bleeding complications or nondesirable anti-inflammatory effects [5].

For distinguishing acute from chronic DVT, or more generally to estimate the age of a thrombus, one intuitive approach is to determine the mechanical property of the blood clot. This is a realistic assumption since blood clots harden over time due to the modification of their intrinsic organization and composition [6]. To pursue this objective, static (or quasi-static) elastography imaging has been proposed as a noninvasive tool to map the strain or normalized elastic modulus of a thrombus from its deformation due to a uniform external compression. Ex vivo and in vivo, the relative elasticity of animal blood clots increases with age [7]-[10]. The same technique was also applied on a population of 54 patients to distinguish acute from chronic DVT using a strain parameter defined as the ratio of the strain within the clot to that of the surrounding tissue [11]. However, such an approach cannot assess quantitative elasticity maps because of uncertainty on the mechanical stress distribution within the probed medium [12]. In addition, it is very challenging to perform such evaluations in the presence of fluid-structure coupling (i.e., a partial clot). These techniques also exhibit low inter and intra-operator reproducibility and a restricted sensitivity range.

To avoid the limitations of static elastography, dynamic elastography (DE) imaging approaches were introduced. The technique involves the generation of low frequency (typically 10-3000 Hz) harmonic or transient shear waves, which propagate into the probed medium, and the tracking of these waves with an ultrafast ultrasound scanner (B-mode [13] or Doppler mode [14]), by MRI [15] or by optic-based techniques [16]. Because tissue elasticity can be deduced from the shear wave velocity or wavelength parameters, $\mathrm{DE}$ can quantitatively map stiffness of the medium. This method allowed characterizing mechanical properties of a wide variety of organs or diseases [17]-[22] but only two studies were proposed to assess, in 
vitro, viscoelastic properties of blood during and after clotting [23], [24]. Due to the 1-D formulation problem and the chosen shear wave generation technique, only one absolute clot elasticity and one biased viscosity value at a single excitation frequency could be calculated using the proposed method [23]. In contrast, the technique introduced in [24] could assess the complex shear modulus (both elastic and viscous parts without any assumption about the viscoelastic law of the blood clot) in a wide frequency range (from 50 to $160 \mathrm{~Hz}$ ), but such an approach is not applicable in vivo because the characterization was performed on a large parallelepiped volume of blood, assuming a homogeneous material.

More recently, we proposed a new concept based on the mechanical resonance of a cylindrical soft heterogeneity to quantitatively assess its viscoelasticity, with sub-objectives consisting of increasing the measurement sensitivity by accentuating vibrations and improving segmentation of the heterogeneity by increasing its mechanical contrast [25]. The proposed shear wave induced resonance elastography (SWIRE) method is based on the phenomenon of confined inclusion resonance induced by

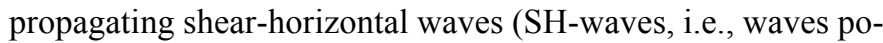
larized parallel to the long axis of the heterogeneity). Using such a configuration, the confined cylindrical heterogeneity is forced into mechanical resonance at particular frequencies. A great advantage of this approach is that the corresponding resonance spectrum (resonance frequencies) is strongly dependent of the inclusion diameter and viscoelasticity. Thus, one can retrieve the viscoelastic parameters of the confined inclusion by formulating an appropriate inverse problem involving a theoretical model. However, the proposed geometry (a unique cylinder surrounded by a harder material) and imaging strategy (3-D acquisitions using a set of monochromatic waves) involved in the original SWIRE technique [25] need to be reformulated and adapted for DVT imaging in a clinical context.

In this study, we propose the DVT-SWIRE technique as an imaging modality capable of characterizing quantitative elasticity of mimicking and real thrombi. Specifically, after introducing the data processing and validating the forward problem that integrates the finite element method (FEM), the inverse problem formulation is tested in an in vitro study including realistic mimicking DVT phantoms (partial and total occlusions). Assessments are compared to "reference" measurements to evaluate the accuracy and sensitivity of the DVT-SWIRE technique. Furthermore, ex vivo phantoms containing thrombosed inferior vena cava and right common iliac veins of a porcine model are mechanically characterized. The paper concludes with a discussion of the results and the applicability for in vivo imaging.

\section{MATERIALS AND MethodS}

\section{A. In Vitro Phantoms, Experimental Setup, and Data Acquisition}

Realistic phantoms composed of a fluid-filled arterial conduit and a mimicking acute total or partial venous clot were built for in vitro experiments. They consisted of $17 \times 16 \times 12 \mathrm{~cm}^{3}$ parallelepiped blocks (mimicking leg muscles) containing two inclusions having similarity to real tissues: a completely [Fig. 1(a)]

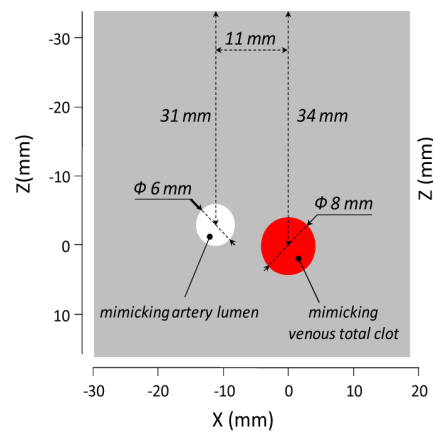

(a)

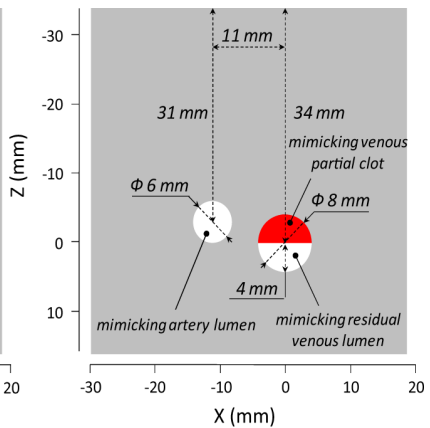

(b)
Fig. 1. Schematic drawing illustrating the geometries of in vitro phantoms with mimicking total (a) or partial (b) clots. (Color online).

or a partially [Fig. 1(b)] occluded 8-mm-diameter and 120-mmlong inclusion mimicking a venous thrombosis, and a 6-mm-diameter and 120-mm-long cylindrical cavity filled with degassed water representing the healthy artery. Following the protocol described in [26], the materials involved in the phantom fabrication were composed of a mixture of agar $(1 \%, 2 \%$, or $3 \%$, Sigma Chemical, number A-6924, Saint Louis, MO, USA) that also provided ultrasound scatterers, gelatin $(2 \%, 3 \%$, or $4 \%$, Sigma Chemical, number G-2500 Type A from porcine skin) forming the structural matrix and water. Tested concentrations of each phantom component are summarized in Table I. To simulate different acute clot maturity (i.e., stiffness), nine phantoms (seven with total clots, two with partial clots) were made and divided into three groups: very soft (phantom \#1), medium soft (phantoms \#2-\#7), and soft (phantoms \#8 and \#9).

We investigated two exciting wave types generated in close proximity of the scanned region (radial distance from the mimicking clot at $25-32 \mathrm{~mm}$; see the vibrating devices on the right side of the phantom in the $x-y$ plane of Fig. 2). We used plane wavefronts as in [26] and [27] and cylindrical wavefronts as already proposed by a few groups [28]-[30]. For both wavefronts, the acquisition protocol consisted of generating transient (six oscillations modulated with a Blackman temporal window) shear waves at frequencies from 45 to $205 \mathrm{~Hz}$ with steps of $20 \mathrm{~Hz}$. The use of short-duration vibrations has also the advantage of avoiding wave reflections from boundaries with proper temporal windowing.

As illustrated in Fig. 2 (left panel), the experimental setup for the plane wavefront configuration consisted of a $14 \times 15 \mathrm{~cm}^{2}$ aluminum rigid plate positioned at the phantom surface and vibrating parallel to the inclusion's long axis (y-axis) to generate shear-horizontal plane waves (SH-waves). This vibration was transmitted to the plate by means of a mini-shaker (type 4810, Brüel\&Kjær, Nærum, Denmark) powered by a function generator (model 33250A, Agilent, Palo Alto, CA, USA) configured using the general-purpose interface bus (GPIB). The excitation signal was amplified with a power amplifier (low frequency amplifier, type 2706, Brüel\&Kjær). The local tissue motion was tracked using a $10 \mathrm{MHz}$ linear probe (L14-5/38, Ultrasonix) connected to a Sonix RP scanner (Ultrasonix Medical Corporation, Burnaby, BC, Canada), and located at the upper phantom surface (see Fig. 2). In order to propose a scanning configuration adapted to the clinical context of DVT cross-sectional 
TABLE I

in Vitro Phantom Composition (AgaR-Gelatin Concentrations), MEAn Viscoelasticity (Elasticity $\mu$ and Viscosity $\eta$ ), and CoRRESPONDing StANDARD DEVIATIONS MEASURED With THE RHEOSPECTRIS REFERENCE INSTRUMENT FOR THE DifFERENT MATERIALS MIMICKING THE CLOT OR THE LEG Muscles. The Materials Were Classified as Very Soft, Medium Soft, and Soft. For Given Agar-gelatin Mixture Concentrations, the

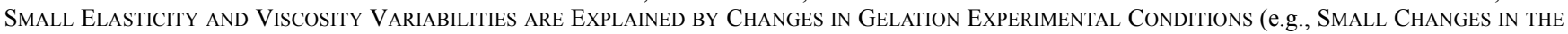
Ambient Temperature). To Assure Similar Conditions to Compare DVT-SWIRE and RheoSpectris Elasticity Measures, THE RheoSpeCtris

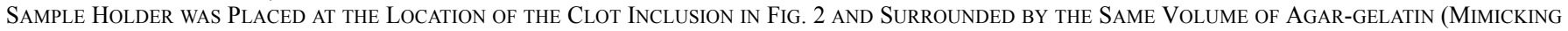
Muscles) to Assure Comparable Gelation. The Sample Holder Containing the Agar-gelatin Gel was Removed From the Phantom for THE MEASUREMENT IN THE RHEOSPECTRIS INSTRUMENT

\begin{tabular}{|c|c|c|c|c|c|c|c|c|}
\hline \multirow[t]{2}{*}{$\begin{array}{l}\text { Phantom } \\
\text { number }\end{array}$} & \multirow[t]{2}{*}{$\begin{array}{c}\text { Softness } \\
\text { level }\end{array}$} & \multirow[t]{2}{*}{$\begin{array}{l}\text { Clot } \\
\text { type }\end{array}$} & \multicolumn{3}{|c|}{$\begin{array}{c}\text { Mimicking clot } \\
\text { (inclusion) } \\
(n=3)\end{array}$} & \multicolumn{3}{|c|}{$\begin{array}{l}\text { Mimicking leg muscles } \\
\text { (surrounding medium) } \\
\qquad(n=3)\end{array}$} \\
\hline & & & $\begin{array}{c}\text { Agar (A) } \\
\text { Gelatin (G) }\end{array}$ & $\begin{array}{c}\mu_{\text {Reference }} \\
(\mathrm{Pa})\end{array}$ & $\begin{array}{c}\eta_{\text {Reference }} \\
\text { (Pa.s) }\end{array}$ & $\begin{array}{c}\text { Agar (A) } \\
\text { Gelatin (G) }\end{array}$ & $\begin{array}{c}\mu_{\text {Reference }} \\
(\mathrm{Pa})\end{array}$ & $\begin{array}{c}\eta_{\text {Reference }} \\
\text { (Pa.s) }\end{array}$ \\
\hline 1 & Very Soft & Total & $\begin{array}{l}A: 1 \% \\
G: 2 \%\end{array}$ & $406 \pm 3$ & $0.092 \pm 0.003$ & $\begin{array}{l}A: 3 \% \\
G: 4 \%\end{array}$ & $17326 \pm 1660$ & $1.090 \pm 0.240$ \\
\hline 2 & \multirow{6}{*}{$\begin{array}{l}\text { Medium } \\
\text { Soft }\end{array}$} & Total & $\begin{array}{l}A: 1 \% \\
G: 3 \%\end{array}$ & $2020 \pm 29$ & $0.163 \pm 0.025$ & $\begin{array}{l}A: 3 \% \\
G: 4 \%\end{array}$ & $18440 \pm 989$ & $0.808 \pm 0.061$ \\
\hline 3 & & Total & $\begin{array}{l}A: 1 \% \\
G: 3 \%\end{array}$ & $1927 \pm 88$ & $0.162 \pm 0.027$ & $\begin{array}{l}A: 3 \% \\
G: 4 \%\end{array}$ & $15466 \pm 31$ & $0.673 \pm 0.014$ \\
\hline 4 & & Total & $\begin{array}{l}A: 1 \% \\
G: 3 \%\end{array}$ & $1829 \pm 27$ & $0.146 \pm 0.020$ & $\begin{array}{l}A: 3 \% \\
G: 4 \%\end{array}$ & $14836 \pm 751$ & $0.685 \pm 0.018$ \\
\hline 5 & & Total & $\begin{array}{l}A: 1 \% \\
G: 3 \%\end{array}$ & $1910 \pm 45$ & $0.162 \pm 0.023$ & $\begin{array}{l}A: 3 \% \\
G: 4 \%\end{array}$ & $16247 \pm 590$ & $0.479 \pm 0.031$ \\
\hline 6 & & Total & $\begin{array}{l}A: 1 \% \\
G: 3 \%\end{array}$ & $1673 \pm 31$ & $0.130 \pm 0.013$ & $\begin{array}{l}A: 3 \% \\
G: 4 \%\end{array}$ & $15393 \pm 766$ & $0.768 \pm 0.126$ \\
\hline 7 & & Partial & $\begin{array}{l}A: 1 \% \\
G: 3 \%\end{array}$ & $2383 \pm 90$ & $0.171 \pm 0.026$ & $\begin{array}{l}A: 3 \% \\
G: 4 \%\end{array}$ & $20196 \pm 300$ & $0.766 \pm 0.065$ \\
\hline 8 & \multirow{2}{*}{ Soft } & Total & $\begin{array}{l}A: 2 \% \\
G: 3 \% \\
\end{array}$ & $3133 \pm 217$ & $0.29 \pm 0.059$ & $\begin{array}{l}A: 3 \% \\
G: 4 \%\end{array}$ & $12409 \pm 228$ & $0.680 \pm 0.012$ \\
\hline 9 & & Partial & $\begin{array}{l}A: 2 \% \\
G: 3 \%\end{array}$ & $3561 \pm 69$ & $0.206 \pm 0.011$ & $\begin{array}{l}A: 3 \% \\
G: 4 \%\end{array}$ & $22835 \pm 1896$ & $1.490 \pm 0.190$ \\
\hline
\end{tabular}

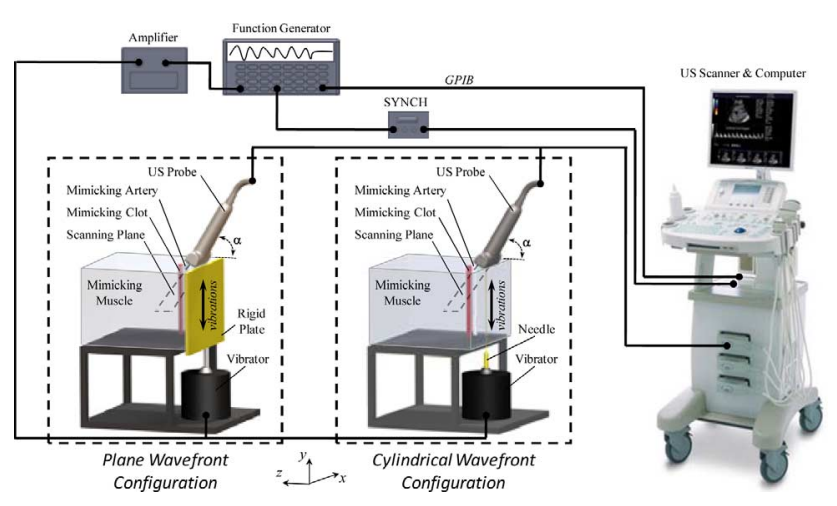

Fig. 2. In vitro experimental set-up used to generate and track plane (rigid plate vibration) or cylindrical (needle vibration) shear waves to perform shear wave induced resonance elastography imaging for deep venous thrombosis application (DVT-SWIRE). An ultrasound scanner properly synchronized with the shearing source using a dedicated electronic circuit (SYNCH) allowed the acquisition of high frame rate RF sequences. (Color online).

image acquisitions, and considering that shear waves were polarized following the $y$-direction and propagated in the $z$-direction, the probe was positioned with an angle $\alpha$ at $65^{\circ}$ from the surface to acquire radio-frequency (RF) sequences. This probe orientation allowed tracking of shear wave displacement projection along the 2-D scanning plane. To follow shear wave propagation, an electronic circuit (SYNCH) was designed to properly synchronize the first RF image acquisition with the low shear wave pulsation for retrospective RF image reconstructions [24]-[26]. This allowed the offline reconstruction of RF sequences (width $\times$ depth $\times$ time of $38-\mathrm{mm} \times 80-\mathrm{mm} \times 260-\mathrm{ms}$ ) at a high frame rate of 3850 images/s.

For the cylindrical wavefront configuration, the experimental arrangement was equivalent to the above-mentioned one except that shear waves were generated by the $y$-direction vibration of a needle (18 Gauge, $1.3-\mathrm{mm}$ outer diameter, $90-\mathrm{mm}$ long) located in proximity of the scanned region of interest (see Fig. 2, right panel). Such a configuration roughly simulated the radiation pressure on a line segment, as generated in the case of an ultrasonic "pushing" beam along the $y$-axis [31].

\section{B. DVT Animal Model and Ex Vivo Phantoms}

In the proposed protocol, one male Landrace pig $(70 \mathrm{~kg})$ was first anaesthetized with a perfusion of propofol $(0.1-0.2 \mathrm{mg} / \mathrm{kg} /$ $\mathrm{min}$ ) and placed in the supine position. During the entire experiment, the pig was ventilated and anesthesia was maintained with a $2 \%$ isoflurane air mix. The surgery consisted of a skin incision at the position of the inferior vena cava located $1 \mathrm{~cm}$ below the renal veins. A segment of the vena cava was surgically dissected along about $1 \mathrm{~cm}$ and tied off with a 7-mm-diameter spacer (equivalent to around $50 \%$ of the vein lumen diameter). Additionally, the vena cava was pinched with a surgical instrument all along the surgical segment and upstream to the lumen narrowing to generate local endothelial damage to amplify the formation and development of thrombosis. A segment 
of the left common femoral vein was then dissected along about $1 \mathrm{~cm}$ and cannulated with a catheter for the infusion of bovine thrombin (total of $500 \mathrm{IU}$, number T4648, Sigma-Aldrich Inc., St. Louis, MO, USA) at a rate of $12.5 \mathrm{IU} / \mathrm{min}$ during $40 \mathrm{~min}$. After 20 min of thrombin injection, the vena cava was completely compressed to induce complete blood stasis. Three hours after the beginning of surgery, the pig was euthanized and the subsequent autopsy consisted of the extraction of the entire venous segment of interest. The inferior vena cava, the iliac bifurcation and common iliac veins, which were partially occluded, were dissected out and removed en bloc for ex vivo experiments. The specimen was then separated into three segments that were immediately cast in agar-gelatin blocks identical to those employed for in vitro experiments: i.e., the inferior vena cava segment and both common iliac segments forming the bifurcation. The ex vivo measurements were performed $24 \mathrm{~h}$ postsurgery. All procedures were approved by the animal care committee of the Centre de Recherche du Centre Hospitalier de l'Université de Montréal in accordance with the Canadian Council on Animal Care guidelines and also conformed with the guide for the care and use of laboratory animals of the USA National Institutes of Health (assurance number A5377-01).

\section{FEM Simulations}

Shear wave propagation has been simulated by the finite element method (FEM). This has been performed using a commercial FEM software (COMSOL Inc., ver. 3.3, Burlington, MA, USA). Materials constituting the media were assumed isotropic, locally homogeneous and linear viscoelastic. For simplification, the wave propagation problem was considered as 2-D and formulated in a Cartesian system of coordinates. The assumption of 2-D simulation is valid regarding the experimental setting ( $\mathrm{SH}$ waves and a cylindrical inclusion geometry) and by considering that the displacement field does not depend on the $y$ coordinate. Moreover, because of the mechanical configuration we selected, it is important to note that there was no mode conversion of the SH waves. The FEM method consisted of solving the Navier differential equation. By assuming harmonic time dependence and omitting the term $e^{i \omega t}$, this equation is expressed as follows:

$$
\rho \omega^{2} \mathbf{U}+(\lambda+2 G) \nabla(\nabla \cdot \mathbf{U})-\mu \nabla \times(\nabla \times \mathbf{U})=0
$$

where $\boldsymbol{U}(x, y)$ is the displacement vector field, $\rho$ is the material density, $\lambda$ and $G$ are the first complex material Lamé viscoelastic coefficient and the complex shear modulus, respectively, that depend on the spatial location $(z, x)$. The spatial distribution of the shear modulus $G=G^{\prime}+i G^{\prime \prime}$ coefficient, with $G^{\prime}$ and $G^{\prime \prime}$ the storage and loss moduli, was used to model local mechanical heterogeneities. Moreover, the viscoelastic behavior of materials was considered to be governed by the Kelvin-Voigt model defined as $G=\mu+i \omega \eta$, where $\mu$ and $\eta$ are the elastic and viscous parameters. This rheological law was previously proven to well predict the dynamic behavior of agar-gelatin materials [27]. This model is also adequate to predict the elasticity of blood clots (in [24], the Zener rheological model was proposed for blood clots but for the conditions of the present study, that model can be approximated by the Kelvin-Voigt equation, which is simpler; see the inverse problem formulation described later in Section II-D, the viscosity of ex vivo blood clots was not determined in this study).

In the simple case, for example, of a centered circular mechanical heterogeneity of radius $R$ representing the total clot, the complex shear modulus distribution had the following form:

$$
\begin{aligned}
& G(z, x) \\
& \quad= \begin{cases}\mu_{\text {inclusion }}+i \omega \eta_{\text {inclusion }}, & \text { if }\left(z^{2}+x^{2}\right)<R^{2} \\
\mu_{\text {surrounding }}+i \omega \eta_{\text {surrounding }}, & \text { if }\left(z^{2}+x^{2}\right) \geq R^{2}\end{cases}
\end{aligned}
$$

where $\mu_{\text {inclusion }}+i \omega \eta_{\text {inclusion }}$ and $\mu_{\text {surrounding }}+i \omega \eta_{\text {surrounding }}$ are the complex viscoelastic shear moduli of the heterogeneity (blood clot) and of the surrounding medium, respectively. In the presence of more complex geometries corresponding to $e x$ vivo experiments, the different boundaries (blood clot, arterial and venous wall boundaries, agar-gelatin phantom mimicking leg muscles) were known or manually segmented from experimental images (B-mode and wave displacement maps) and integrated into the FEM model.

Two types of shear wave sources were modeled depending on the wavefront configuration. For plane waves, at each node of one boundary of the domain was applied a constant displacement amplitude, i.e., the vibration amplitude of the rigid plate with no attenuation by the medium, whereas in the case of cylindrical wavefronts, the rigid needle motion was introduced at each node of the mesh contained within a volume defining a 1.3-mm circle (i.e., the needle diameter). For both shear wave vibration types, the artery depicted in Fig. 2 was simulated by a circle with an inner surface that was considered to be unpressurized, i.e., with no stress at the boundary (as performed experimentally). This modeling assumption was sufficient to simulate the absence of shear wave propagation within the mimicked artery filled with water.

To avoid undesirable reflections of shear waves on the free boundaries of the meshed volume, we introduced strongly attenuating artificial layers surrounding the mimicking agar-gelatin leg muscle. This was achieved by imposing to the shear modulus in these layers a real part equal to $\mu_{\text {surrounding }}$ (to insure a continuity of the mechanical impedance with the surrounding medium) and an imaginary part increasing exponentially with the thickness of the attenuating layer. FEM simulations were performed in the frequency domain. Element sizes were equal to a maximum of a third of the shear wavelength $\lambda$ to ensure appropriate FEM sampling. After calculating the solution, the mapping of the complex stationary shear wave displacement within the domain was extracted with the Matlab software (ver. 6.5, MathWorks Inc., Natick, MA, USA) for further postprocessing.

\section{Data Postprocessing and Inverse Problem Formulation}

As illustrated in Fig. 3, data postprocessing consisted of the following steps.

1) The spatio-temporal displacements into the medium $(u(z, x, t))$ induced by transient shear waves were computed using a normalized cross-correlation (NCC) algorithm applied to the RF temporal sequences $\left(I_{\mathrm{RF}}(z, x, t)\right)$. This algorithm was efficiently implemented using a graphic processing unit (GPU) to speed-up the processing time needed for future real-time applications. 


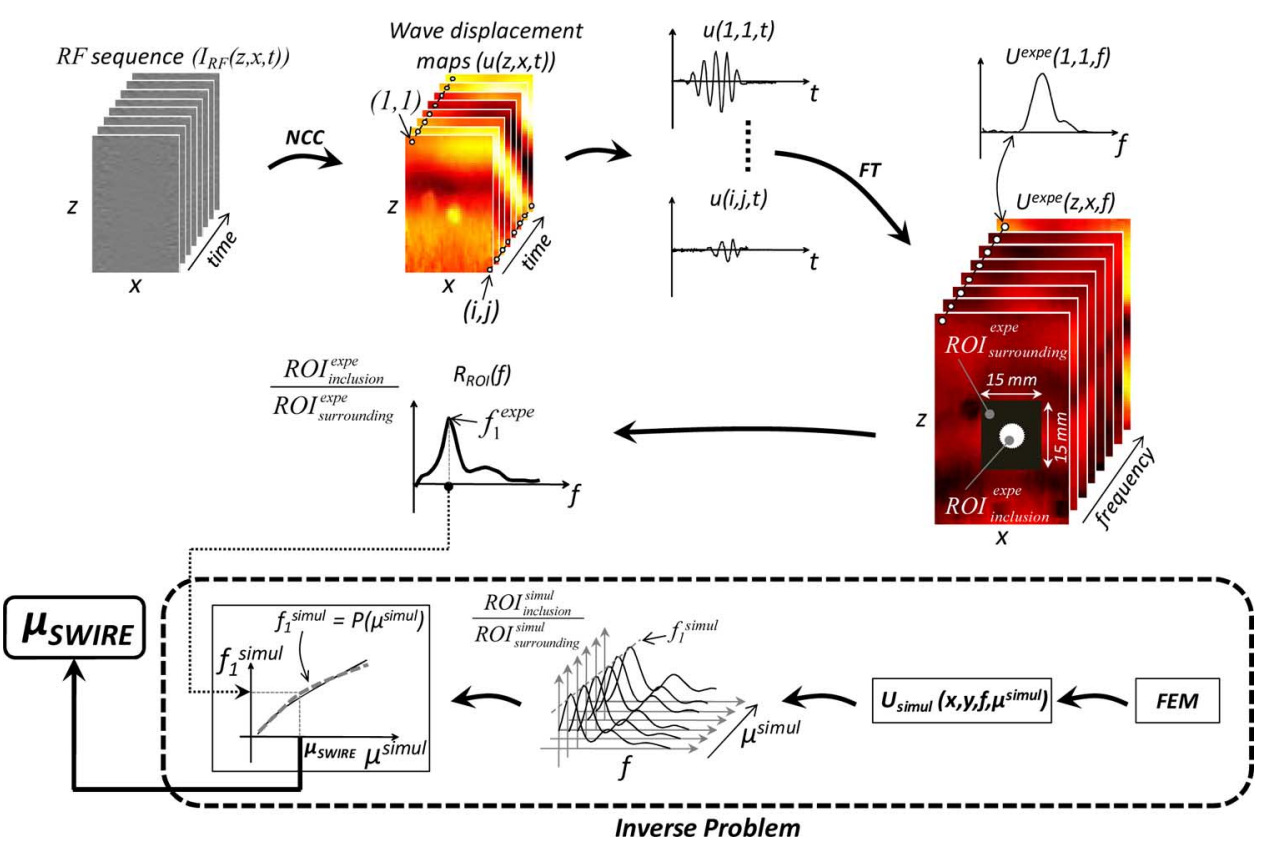

Fig. 3. Block-diagram of data processing for the estimation of the inclusion elasticity ( $\left.\mu_{\text {SWIRE }}\right)$ using the DVT-SWIRE technique. This integrates the processing of experimental data and the formulation of the inverse problem (see text for details). (Color online)

2) For each pixel of the time-varying displacement waveform, a Fourier transform (FT) was computed and the complex amplitudes, in a spectral window of $100 \mathrm{~Hz}$ (centered at the generated frequency with a frequency step of $0.1 \mathrm{~Hz}$ ), were used to form a matrix containing the absolute part of the stationary displacement fields as a function of frequency $\left(U^{\operatorname{expe}}(z, x, f)\right)$.

3) For each frequency of $U^{\operatorname{expe}}(z, x, f)$, the average values within two regions defined by the masks $\mathrm{ROI}_{\text {surrounding }}$ (15-cm squared region) and $\mathrm{ROI}_{\text {inclusion }}$ (within the inclusion boundary) representing the surrounding material and the inclusion, respectively, served to calculate the spectrum of their ratio as a function of frequency $\left(R_{\mathrm{ROI}}(f)\right)$

$$
R_{\mathrm{ROI}}(f)=\frac{\sum_{z, x \in \mathrm{ROI}_{\text {inclusion }}^{\text {expe }}}\left|U^{\operatorname{expe}}(z, x, f)\right|}{\sum_{z, x \in \mathrm{ROI}_{\text {surpeunding }}^{\text {expe }}}\left|U^{\operatorname{expe}}(z, x, f)\right|} .
$$

In this equation, $R_{\mathrm{ROI}}(f)$ is a simple representation of the contrast between the motion of the inclusion and that within a region surrounding the inclusion. This spectral ratio allowed the identification of the experimental resonance frequency $f_{1}^{\text {expe }}$ used in the inverse problem formulation.

4) The inverse problem was formulated by employing a parametric approach. FEM simulations were used to calculate the stationary fields $U^{\text {simul }}$ for different frequencies $f$ and inclusion elastic moduli $\mu^{\text {simul }}$. The first simulated resonance frequency $\left(f_{1}^{\text {simul }}\right)$ as a function of the elastic modulus $\left(\mu^{\text {simul }}\right)$ was extracted from the corresponding simulated spectral ratio of (3) (same equation with the subscripts simul instead of expe) by indentifying the frequency of the peak amplitude. The evolution of $f_{1}^{\text {simul }}$ as a function of $\mu^{\text {simul }}$ was then fitted with a fifth-order polynomial function $P$ to precisely retrieve the researched phantom inclusion elasticity $\mu_{\mathrm{SWIRE}}$, using the following minimization problem:

$$
\mu_{\text {SWIRE }}=\arg \min \left|P\left(\mu^{\text {simul }}\right)-f_{1}^{\text {expe }}\right| .
$$

For this minimization, the elasticity and viscosity of the mimicked leg muscles and viscosity of the agar-gelatin clot inclusions were fixed to values listed in Table I (for the ex vivo study, the viscosity of the blood clot was assigned to a value of $0.2 \mathrm{~Pa} \cdot \mathrm{s}$ ).

\section{E. Effect of Segmented Boundary Variations on the Reliability of the Inverse Problem}

The inverse problem formulation applied to ex vivo phantom data was validated by considering variations in the segmented boundary of the porcine inferior vena cava thrombus. The segmented thrombus area was first reduced or increased by $-20 \%$ to $32 \%$, while maintaining the original shape. To determine the effect of boundary variability, normally distributed random noise was applied to the 52 equally distant control points defining the segmented contour. The signal-to-noise ratio (SNR) was calculated as

$$
\mathrm{SNR}=20 \log _{10}\left(\frac{P_{\text {contour }}}{P_{\text {noise }}}\right)
$$

where $P_{\text {contour }}$ corresponds to the initial positions of the segmented boundary and $P_{\text {noise }}$ is the root mean square of the applied shifts.

\section{F. Effect of the Surrounding Medium Viscoelasticity A Priori on the Reliability of the Inverse Problem}

An additional validation of robustness was performed to determine the relative importance of the surrounding medium viscoelasticity a priori on the inverse problem applied to the ex vivo 
phantom geometry. For this purpose, the resonance frequency of the thrombus was simulated and the inverse problem in Fig. 3 was determined for surrounding medium viscoelasticity varying between $\pm 40 \%$ of its initial value given in Table I (phantom \#9).

\section{G. Viscoelastic Characterization With a Reference Instrument}

During the preparation of phantoms, the same gel mixtures used in the fabrication of the inclusion and surrounding materials were poured into six (three for each material) 9-mm-diameter and 70-mm-long cylindrical plastic test tubes and then analyzed with the RheoSpectris viscoelastic spectroscope (model C-0, Rheolution Inc., Montreal, QC, Canada), defined as reference mechanical tests [32]. From the complex shear modulus (storage and loss moduli) measured by the instrument over frequencies ranging from 10 to $1000 \mathrm{~Hz}$, a rheological model fitting toolbox implemented in the RheoView software (ver. 1.1, Rheolution Inc.) was employed to assess the viscoelastic parameters defining the simple Kelvin-Voigt model. The elasticity parameter $\mu$ was calculated from the average value of $G^{\prime}$ over the $10-1000 \mathrm{~Hz}$ frequency range as

$$
\mu=\overline{G^{\prime}}
$$

whereas the viscous parameter $\eta$ was given by the slope of the linear regression applied on the Voigt model imaginary part

$$
\eta=\arg \min _{\eta}\left\|G^{\prime \prime}-\omega \eta\right\|_{2}^{2} .
$$

\section{RESULTS}

\section{A. Validation of Forward Problems}

In this section, we first present the validation of the forward problems for the plane and cylindrical wavefront configurations for both total (phantom \#2) and partial (phantom \#7) mimicking clots. For all simulated FEM data, the viscoelasticity of materials (inclusion and surrounding medium) was given by reference measurements obtained with the RheoSpectris instrument (see data in Table I).

A B-mode image for the plane wavefront configuration with a total clot, calculated from the log-compressed RF signal envelope, is presented in Fig. 4(a). The real positions and sizes of the modeled arterial lumen and venous total clot are identified on this figure according to the phantom mould geometry. Taking into account the frequency wideband nature of a $125-\mathrm{Hz}$ transient wave, displacement spectra between $105 \mathrm{~Hz}$ and $165 \mathrm{~Hz}$ [Fig. 4(b)] were determined for four measurements within the inclusion [at its center as identified by a white cross symbol in Fig. 4(a)]. This plot depicts higher motion amplitudes at $123.5 \pm 0.35 \mathrm{~Hz}$ [from (3)] and denotes an excellent reproducibility (error of $0.28 \%$ for the peak detection). This equivalent resonance mode was found at a frequency of $121.3 \mathrm{~Hz}$ using the FEM technique. The experimental 2-D normalized stationary displacement field [Fig. 4(c)] calculated at the first resonance frequency $(123.5 \mathrm{~Hz})$ clearly shows an eigenmode, i.e., a strong contrast of motion (equal to 4.4) between the inclusion and the surrounding material. These results were compared with FEM simulated data using a similar configuration (geometry
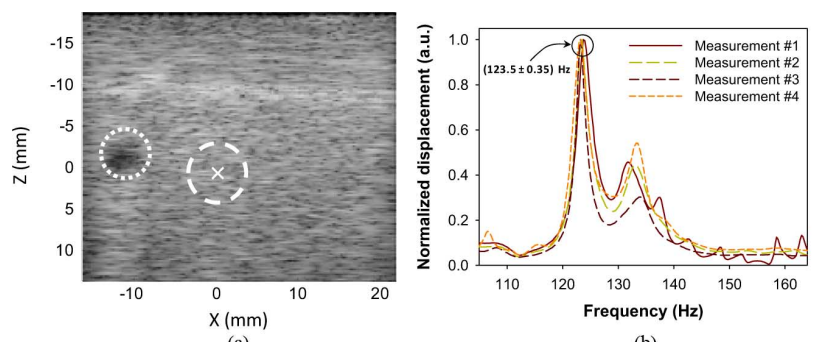

(a)
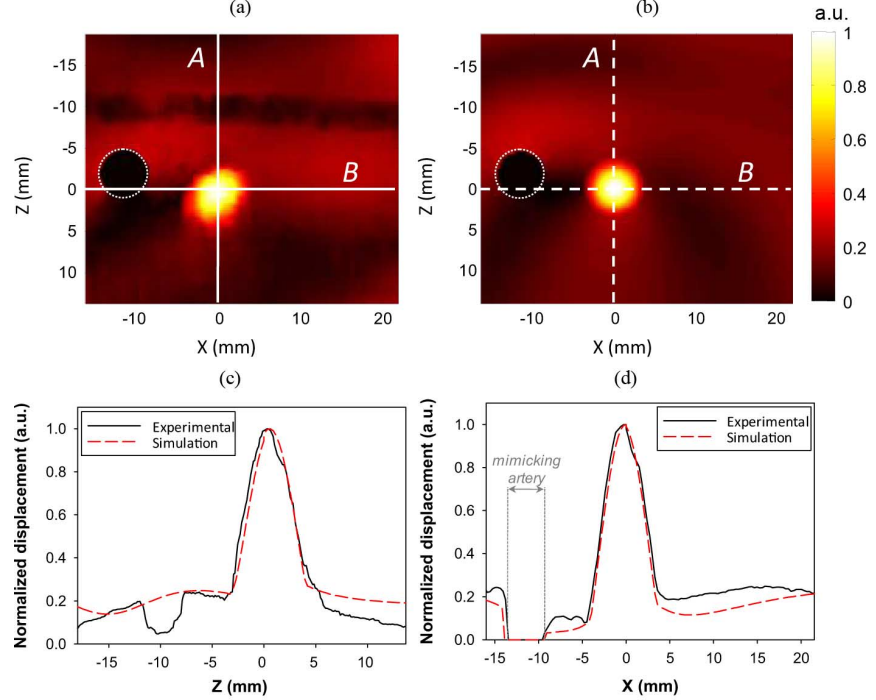

(e)

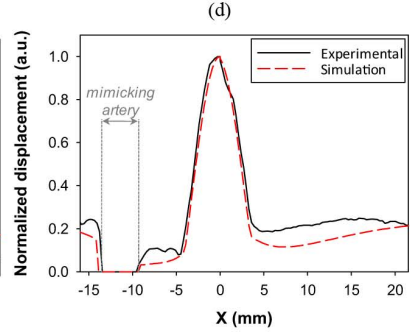

Fig. 4. B-mode image of the probed phantom containing a total inclusion (dashed line) and a water-filled cylindrical cylinder (dotted line) embedded in an agar-gelatin gel (phantom \#2) (a). Displacement spectra within the inclusion [cross in panel (a)] for a $125-\mathrm{Hz}$ plane transient shear wave and for four repeated measurements (b). Corresponding experimental (c) and simulated (d) 2-D normalized stationary displacement maps at the first resonance frequency (123.5 Hz experimental and $121.3 \mathrm{~Hz}$ theoretical) and corresponding profile on line A (axial) in (e) and B (lateral) in (f). (Color online)

and frequency) and are presented in Fig. 4(d). For quantitative comparison, the corresponding 1-D profiles following the $z$-axis and $x$-axis are plotted in Fig. 4(e) and (f), respectively. Equivalent images with the same phantom $\# 2$ but for the cylindrical wavefront configuration were calculated for the vibrating needle positioned at $z=-27 \mathrm{~mm}$ and $x=15 \mathrm{~mm}$ (Fig. 5). Experimental and theoretical resonance frequencies were 122.0 and 122.2 Hz, respectively. Because the imaging plane crosses the $x-y-z$ axes whereas the simulation was done in the $z-x$ plane, the experimental data were registered in the $x-z$ coordinate system by considering the probe orientation (angle $\alpha$ in Fig. 2).

Fig. 6 presents a typical propagation of a $125-\mathrm{Hz}$ transient shear wave into the investigated total clot phantom at different moments. The shearing source, identified by the needle, generated a cylindrical shear wave which, during its propagation through the phantom, was diffracted by the mimicking artery, and amplified by the soft mimicking clot inclusion (see Fig. 6 at times superior to $30.9 \mathrm{~ms}$ ). The wideband spectral energy contained in the transient wave resulted in the inclusion heterogeneity resonance that can clearly be segmented visually [not possible from the B-mode image in Fig. 6]. The same comparisons of FEM with experimental results were also performed for 

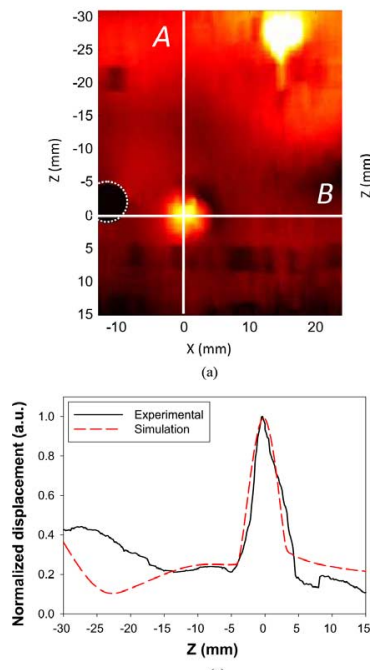

(c)
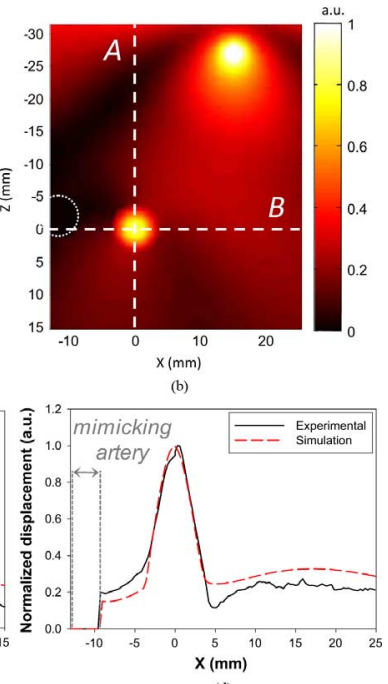

(d)
Fig. 5. Experimental (a) and simulated (b) 2-D normalized stationary displacement maps at the first resonance frequency $(122.0 \mathrm{~Hz}$ and $122.2 \mathrm{~Hz}$ for experimental and simulated data, respectively) for a $125 \mathrm{~Hz}$ cylindrical transient shear wave that propagated within a phantom (phantom \#2) containing a mimicking total clot and a water-filled cylindrical hole (dotted line). The needle is located at positions $z=-27 \mathrm{~mm}$ and $x=15 \mathrm{~mm}$. Corresponding profile on line A (axial) in (c) and B (lateral) in (d). (Color online)

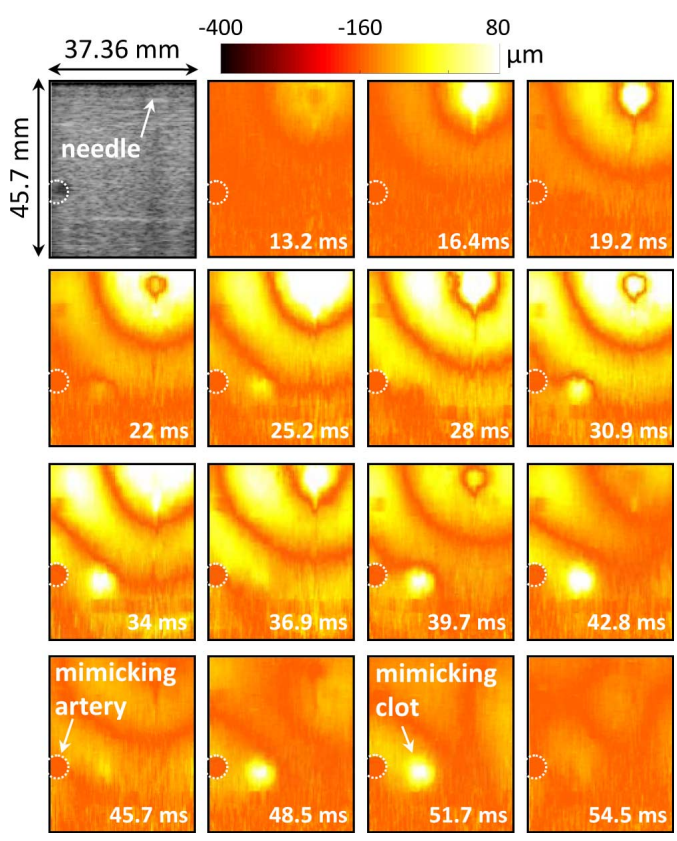

Fig. 6. B-mode image of a mimicking total clot (phantom \#2) made of agargelatin and typical 2-D displacement maps as a function of time for a $125 \mathrm{~Hz}$ cylindrical transient shear wave. (Color online)

the mimicking partial clot, for both plane (Fig. 7) and cylindrical (Fig. 8) transient shear waves at $125 \mathrm{~Hz}$.

Some differences between experimental and FEM results could be observed and identified as artifact signals but did not have any effect on the calculated spectra. Due to the absence of ultrasound absorbers positioned on the phantom's surface, multiple reverberating echo-signals affected RF images and induced decorrelation noise in the calculated shear wave displacement maps. This can be seen in experimental results of Fig. 4(c) and (e) for the $-10 \mathrm{~mm}$ depth $z$ position, in Fig. 5(a)

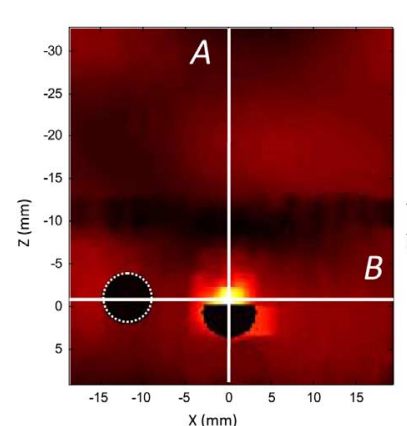

(a)

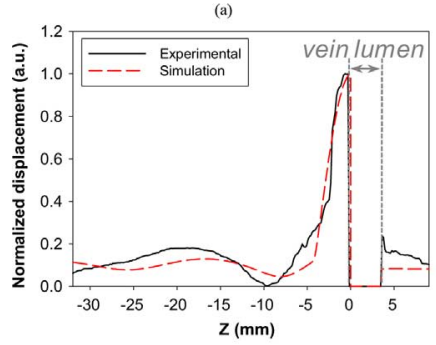

(c)

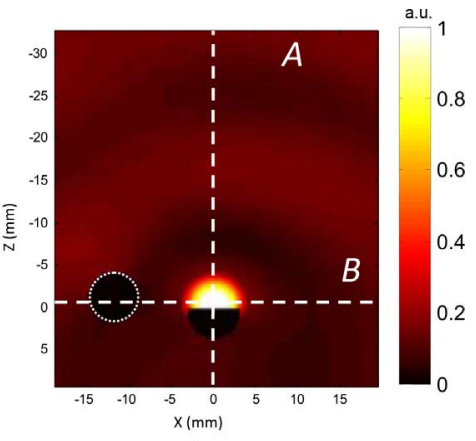

(b)

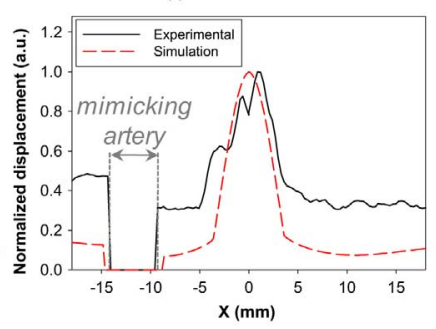

(d)
Fig. 7. Experimental (a) and simulated (b) 2-D normalized stationary displacement maps at the first resonance frequency $(127.0 \mathrm{~Hz}$ experimental and 129.5 $\mathrm{Hz}$ theoretical) for a $125-\mathrm{Hz}$ plane transient shear wave that propagated within a phantom (phantom \#7) containing a mimicking partial clot and a water-filled cylindrical hole (dotted line). The vein lumen was manually segmented. Corresponding profile on line A (axial) in (c) and B (lateral) in (d). (Color online)
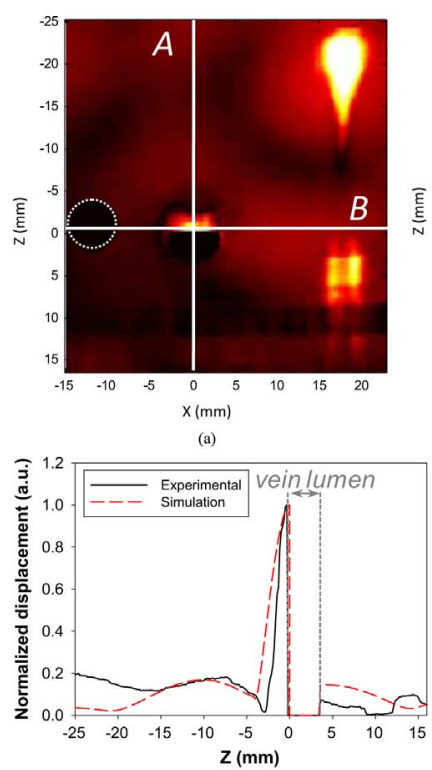

(c)

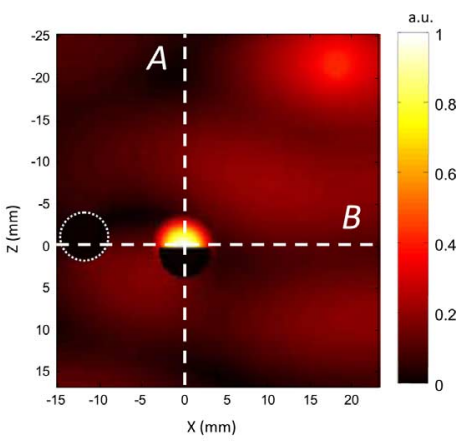

(b)

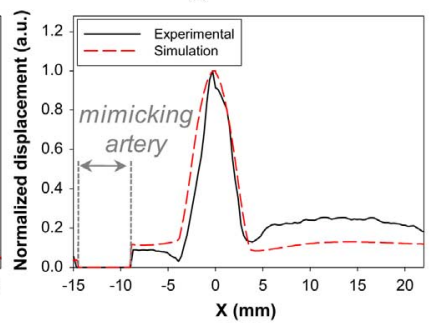

(d)

Fig. 8. Experimental (a) and simulated (b) 2-D normalized stationary displacement maps at the first resonance frequency $(136.0 \mathrm{~Hz}$ experimental and $136.6 \mathrm{~Hz}$ theoretical) for a $125 \mathrm{~Hz}$ cylindrical transient shear wave that propagated within a phantom (phantom \#7) containing a mimicking partial clot and a water-filled cylindrical hole (dotted line). The needle is located at positions $z=-22 \mathrm{~mm}$ and $x=18 \mathrm{~mm}$. The vein lumen was manually segmented. Corresponding profile on line A (axial) in (c) and B (lateral) in (d). (Color online)

and (c) for the $-21 \mathrm{~mm}$ and $6 \mathrm{~mm} z$ positions, in Fig. 7(a) and (c) for the $-10 \mathrm{~mm} z$ position, and in Fig. 8(a) and (c) for the $10 \mathrm{~mm} z$ position. Another ultrasound noise source was induced by the presence of the metallic needle for the cylindrical shear wave generation, which created a shadowing 
TABLE II

First Resonance Frequencies Measured From Experimental and Simulated Data FOR Mimicking Total and Partial Clots, AND FOR PLANE AND CYLINDRICAL WAVEFRONT CONFIGURATIONS IN THE CONTEXT OF THE ForWARD PROBLEM VALIDATION

\begin{tabular}{|c|c|c|c|c|}
\hline \multirow[t]{3}{*}{ Phantom } & \multicolumn{4}{|c|}{ Mimicking total clot } \\
\hline & \multicolumn{2}{|c|}{ Plane wavefront } & \multicolumn{2}{|c|}{ Cylindrical wavefront } \\
\hline & Experimental & Simulation & Experimental & Simulation \\
\hline \multirow[t]{4}{*}{ \#2 } & $123.5 \mathrm{~Hz}$ & 121.3 & $122.0 \mathrm{~Hz}$ & $122.2 \mathrm{~Hz}$ \\
\hline & \multicolumn{4}{|c|}{ Mimicking partial clot } \\
\hline & \multicolumn{2}{|c|}{ Plane wavefront } & \multicolumn{2}{|c|}{ Cylindrical wavefront } \\
\hline & Experimental & Simulation & Experimental & Simulation \\
\hline \#7 & $127.0 \mathrm{~Hz}$ & $129.5 \mathrm{~Hz}$ & $136.0 \mathrm{~Hz}$ & $136.6 \mathrm{~Hz}$ \\
\hline
\end{tabular}

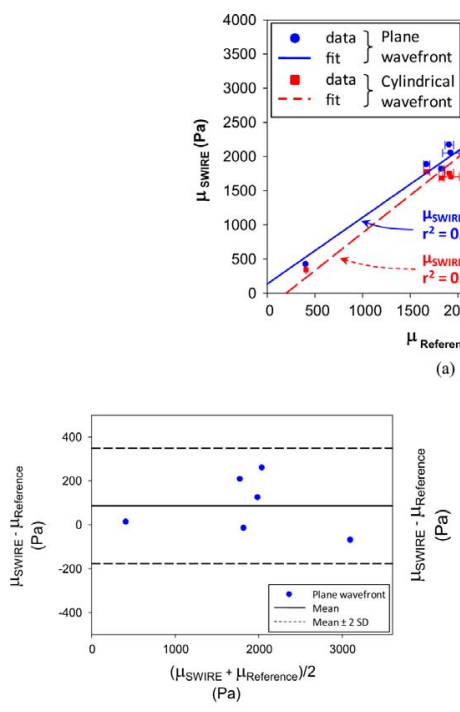

(b)

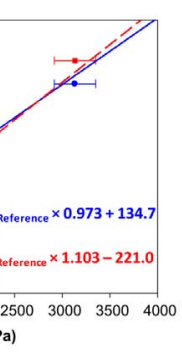

(a)

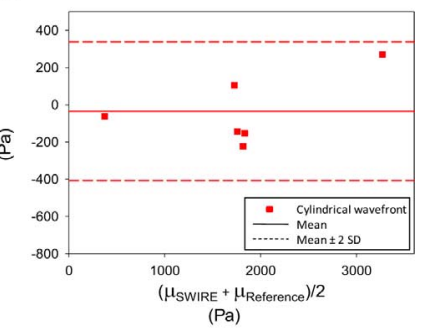

Fig. 9. Linear correlation analysis comparing the elasticity obtained with the DVT-SWIRE technique $\left(\mu_{\mathrm{SWIRE}}\right)$ and the reference measurements for five phantoms (\#1, \#3-6,\#8) when insonified by plane (blue circles) and cylindrical (red squares) shear wavefronts. The standard deviation (SD) is only plotted for the reference measurements (a). The Bland-Altman analysis for the corresponding plane and cylindrical wavefront configurations are plotted in (b) and (c), respectively. In each plot (b and c), the solid horizontal lines correspond to the mean difference and the dashed horizontal lines correspond to the mean \pm 2 SD differences between the elasticities measured with DVT-SWIRE and the reference instrument. (color online)

cone due to strong attenuation (Fig. 5(a) for the $15 \mathrm{~mm}$ lateral $x$ position and Fig. 8 (a) for the $18 \mathrm{~mm}$ lateral $x$ position).

To conclude, the good agreement between experimental and simulated stationary shear wave amplitude distributions reported in Figs. 4, 5, 7, and 8 (both 2-D maps and 1-D profiles), as well as the good matching of first resonance frequencies (see Table II), confirmed the relevance of using such FEM modeling to support the inverse problem formulation. The first eigenmode that presents in-phase motion amplification within the confined cylindrical inclusion is used next to provide a robust inclusion elasticity recovering.

\section{B. Mimicking DVT Quantitative Elasticity Assessment}

Experiments using eight different phantoms (phantoms \#1, and \#3-\#9) served to validate the inverse problem coupled to the simulated (FEM) model for mimicking clot elasticity assessment. The complete data processing and inverse problem solving presented in Section II-D was applied to experimental data. As presented in Fig. 9(a) for the mimicking total clot, linear
TABLE III

COMPARISON OF DVT-SWIRE AND REFERENCE INSTRUMENT ESTIMATES FOR THE Mimicking PARTIAL ClOT AND FOR the CONFIGURATION of Plane AND CYLINDRICAL WAVEFRONTS

\begin{tabular}{|c|c|c|}
\hline \multirow[t]{2}{*}{ Phantom } & $\mu_{\text {SWIRE }}$ & $\mu_{\text {Reference }}$ \\
\hline & \multicolumn{2}{|c|}{ Plane wavefront } \\
\hline \#7 & $2241 \mathrm{~Pa}$ & $2383 \pm 90 \mathrm{~Pa}$ \\
\hline \multirow[t]{2}{*}{$\# 9$} & $2881 \mathrm{~Pa}$ & $3561 \pm 69 \mathrm{~Pa}$ \\
\hline & \multicolumn{2}{|c|}{ Cylindrical wavefront } \\
\hline$\# 7$ & $2506 \mathrm{~Pa}$ & $2383 \pm 90 \mathrm{~Pa}$ \\
\hline$\# 9$ & $2937 \mathrm{~Pa}$ & $3561 \pm 69 \mathrm{~Pa}$ \\
\hline
\end{tabular}

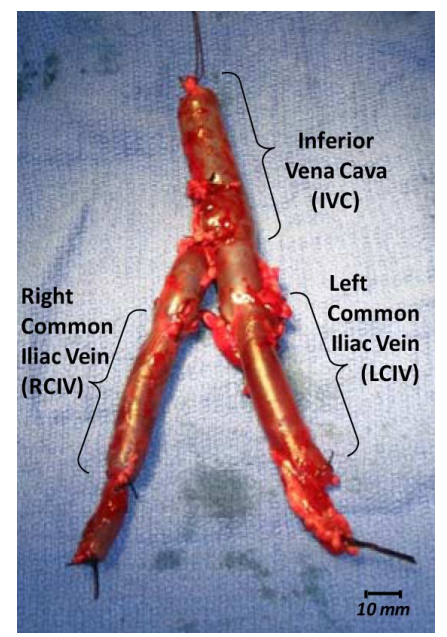

Fig. 10. Picture of the dissected whole venous segment in a pig model of surgically induced DVT. The inferior vena cava (IVC) and the right and left common iliac veins (RCIV and LCIV) were embedded in an agar-gelatin phantom for experimental acquisitions. (Color online)

regression analyses showed that the reference and the calculated inclusion stiffness were highly coincident, with correlation coefficients $r^{2}=0.989$ and 0.986 for the plane and cylindrical wavefront configurations, respectively. The good concordance is also portrayed by the slopes of these two plots that are close to 1 ( 0.973 for plane waves and 1.103 for cylindrical waves), and by the small elasticity biases (134.7 Pa for plane waves and $-221 \mathrm{~Pa}$ for cylindrical waves). The results of the Bland-Altman analysis [33] are also shown on this figure [plane wavefront in panel (b) of Fig. 9 and cylindrical wavefront in panel (c)]. The phantoms with partial clots (\#7 and \#9) were characterized similarly and results are presented in Table III for the plane and cylindrical excitations, and compared with the reference measurements. To summarize, the overall errors of estimation for the 12 measurements with total clots $(9.0 \pm 4.6 \%)$ and the four measurements with partial clots $(9.3 \pm 11.3 \%)$ prove the good accuracy of the proposed method whatever the clot geometry characteristics.

\section{Ex Vivo Porcine Thrombi}

DVT-SWIRE was finally applied to the mechanical characterization of two fresh porcine venous thrombi, one located in the inferior vena cava (IVC) and the other in the right common iliac vein (RCIV). From the three venous segments presented in Fig. 10, we did not analyze the left common iliac vein (LCIV) because multiple small floating clots in liquid blood were noted. 


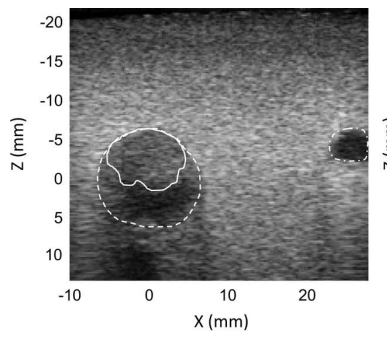

(a)

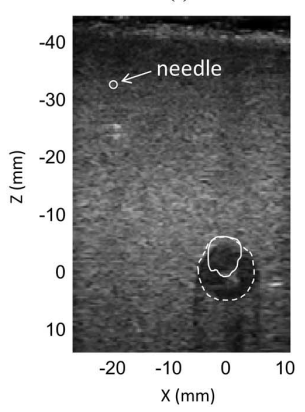

(c)

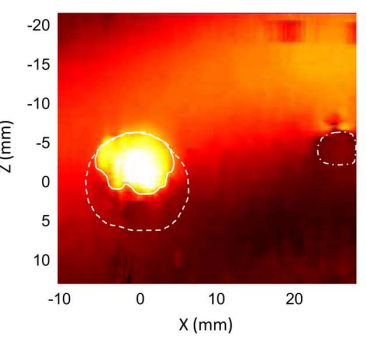

(b)

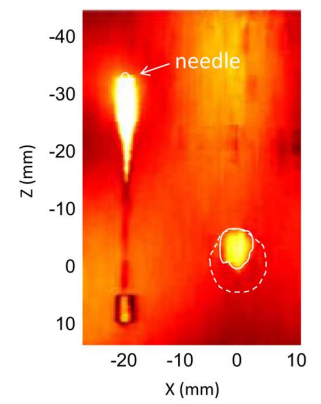

(d)

Fig. 11. B-mode image of the probed phantom made of fresh thrombi formed within the inferior vena cava (IVC) (a) and right common iliac vein (RCIV) (c) segments and manual segmentations of the vessel walls (dotted lines), of the mimicking artery (dot-dashed line), and of the thrombi (solid lines). Corresponding experimental 2-D normalized stationary displacement maps at the first resonance frequency ( $68 \mathrm{~Hz}$ for the IVC and $74 \mathrm{~Hz}$ for the RCIV) for the IVC (b) and the RCIV (d). The shearing source was a $85 \mathrm{~Hz}$ transient plane (b) or cylindrical (d) wave. (color online)

B-mode images of the IVC and RCIV phantoms are presented in Fig. 11(a) and (c), respectively. Manual segmentations of the vessel walls (dotted lines), of the mimicking artery for the IVC phantom (dot-dashed line), and of the venous thrombi (solid lines) are presented on this figure. Panels (b) and (d) of Fig. 11 show the normalized displacement maps at the resonance frequency $\left(f_{1}=68 \mathrm{~Hz}\right.$ for the IVC thrombus and $74 \mathrm{~Hz}$ for the RCIV blood clot) for a transient excitation frequency of $85 \mathrm{~Hz}$. A plane wavefront is displayed for the IVC phantom and a cylindrical wavefront is depicted for the RCIV. For both phantoms, similar results (not shown) were obtained with either the plane or cylindrical wavefront. A good concordance can be noticed between the manually segmented blood clots on B-mode images and resonance display maps in panels (b) and (d).

The first resonance frequency determined by sweeping the transient wave excitation from 45 to $205 \mathrm{~Hz}$ and segmented geometries (i.e., clot, vessel wall boundaries, and phantom dimensions) were introduced in the inverse problem formulation to recover the elasticity of thrombi $\mu_{\text {SWIRE }}$ presented in Fig. 12. Means and standard deviations were calculated for three acquisitions for both thrombi. The mean thrombus elastic moduli were $498 \pm 58 \mathrm{~Pa}$ for the IVC and $436 \pm 45 \mathrm{~Pa}$ for the RCIV (nonsignificant, $p=0.22$, Student t-test). Measurements were reproducible with coefficients of variability of $11.6 \%$ and $10.3 \%$ for IVC and RCIV clots, respectively.

\section{Effect of Segmented Boundary Variations on Ex Vivo IVC Thrombus Elasticity}

Fig. 13(a) presents $\mu_{\text {SWIRE }}$ for the case of changes in segmented surfaces, while maintaining the original shape of the

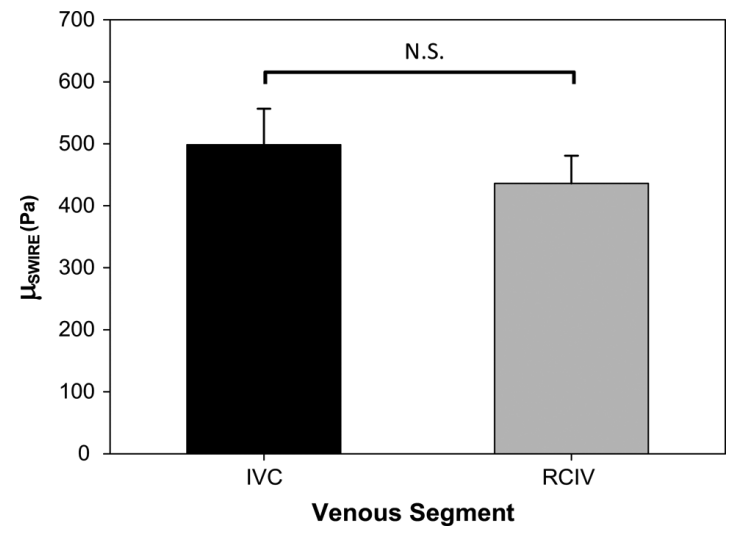

Fig. 12. Elasticity moduli estimated with DVT-SWIRE in the ex vivo study for clots formed within the inferior vena cava (IVC) and the right common iliac vein $(\mathrm{RCIV})(n=3$ per segment). N.S. $=$ not statistically significant.
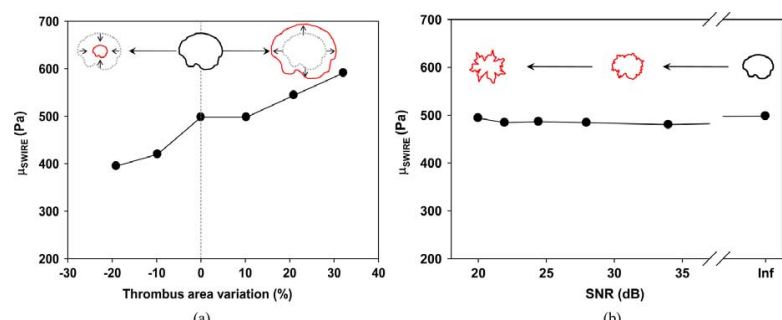

Fig. 13. (a) Elasticity moduli obtained with the DVT-SWIRE technique $\left(\mu_{\text {SWIRE }}\right)$ for the ex vivo thrombus developed in the inferior vena cava (IVC) as a function of an underestimation (ranging from $-20 \%$ to $0 \%$ ) and overestimation (ranging from $0 \%$ to $32 \%$ ) of the segmented thrombus area. (b) Elasticity moduli estimated for a segmented boundary subjected to delineation noise characterized by a signal-to-noise ratio (SNR) between Inf (no noise) and $20 \mathrm{~dB}$. The elasticity for the optimally segmented thrombus is $498 \mathrm{~Pa}$.

IVC thrombus contour. The estimated elasticity of the porcine blood clot progressively varied from $395 \mathrm{~Pa}$ (relative error of $-20.7 \%)$ to $591 \mathrm{~Pa}(+18.6 \%)$ for area variations of $-20 \%$ to $32 \%$. Fig. 13(b) reveals little effect of segmented contour variability on elasticity estimates (maximum relative error of $-3.6 \%$ ). These results demonstrate the robustness of the inverse problem, even if one considers boundary segmentation errors as large as $32 \%$ in area.

\section{E. Effect of Changes in the Surrounding Medium Viscoelasticity on Ex Vivo IVC Thrombus Elasticity}

The ex vivo IVC thrombus geometry was also considered for this further evaluation of robustness. Fig. 14 shows elasticity moduli of the partially occluded thrombus for surrounding medium elasticity varying from 13701 to $31969 \mathrm{~Pa}( \pm 40 \%$ variation with respect to $22835 \mathrm{~Pa}$, see Table I-phantom \#9), at a constant surrounding viscosity of $1.49 \mathrm{~Pa} \cdot \mathrm{s}$. The inverse problem provided similar solutions with the largest error at $2.5 \%$. By varying the surrounding medium viscosity by $\pm 40 \%$ for a constant elasticity of $22835 \mathrm{~Pa}$, no noticeable variations of $\mu_{\text {SWIRE }}$ were noted (data not shown). Again, these results demonstrate the robustness of the proposed elastography method. 


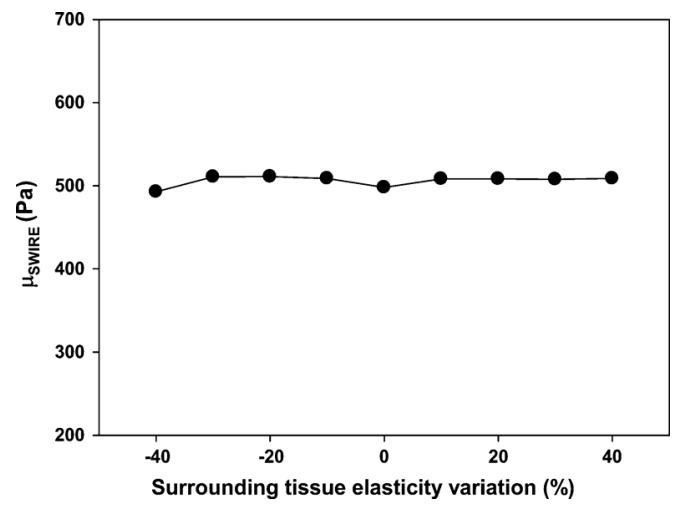

Fig. 14. Elasticity moduli obtained with the DVT-SWIRE technique ( $\left.\mu_{\text {SWIRE }}\right)$ for the ex vivo thrombus developed in the inferior vena cava (IVC) as a function of an underestimation (ranging from $-40 \%$ to $0 \%$ ) and overestimation (ranging from $0 \%$ to $40 \%$ ) of the surrounding tissue elasticity given in Table I (phantom \#9). Similar variations between $\pm 40 \%$ of the surrounding tissue viscosity, also given in Table I, provided no noticeable variations of $\mu_{\text {SWIRE }}$. The expected elasticity of the thrombus is $498 \mathrm{~Pa}$.

\section{DISCUSSION}

The main objective of this study was to propose a novel elastography imaging technique, labeled DVT-SWIRE, to quantitatively assess the elasticity of nine mimicking (agar-gelatin phantoms) and two real venous thrombi (porcine thrombosed vena cava and iliac vein). The estimated elasticity moduli, ranging from 406 to $3561 \mathrm{~Pa}$, were found equivalent to those measured with a reference instrument (Rheospectris). According to the literature, elasticity of small inclusions characterized by dynamic elastography ranged from a few $\mathrm{kPa}$ [25] to some MPa [34], but, as per current knowledge, no studies have been dedicated to small inclusions with such low stiffness (e.g., the 8-mm-diameter cylindrical inclusion and $\mu=406 \mathrm{~Pa}$ for the phantom \#1). This highlights the potential of DVT-SWIRE to detect and characterize incipient thrombosis having low elasticity values.

\section{A. Usefulness of the Vibration Spectral Information Content}

Most methods proposed so far in dynamic elastography used information such as shear wavelength [15], velocity [31], amplitude [35], tissue relaxation [36], etc. but all these parameters are very sensitive to the signal-to-noise ratio because they rely on temporal or spatial derived information. For SWIRE, the frequency information (first resonant frequency) calculated within the inclusion and in a surrounding region is taken into account in an inverse problem, which is expected to be more stable and less sensitive to vibration amplitude noise. In a previous spectral approach proposed by Fatemi and Greenleaf [37], [38], labeled ultrasound-stimulated vibro-acoustography (USVA), they considered very rigid structure resonance (calcification, glass sphere, etc.) due to oscillatory radiating force. By analogy to USVA, SWIRE employs low frequency shear-horizontal waves instead of ultrasound compression waves to induce the resonance of soft inclusions, but the useful information is similar and is described by the evolution of the inclusion motion amplitude as a function of frequency.

In our approach, the vibration spectra within the confined inclusion could be evaluated locally (at one point location) by computing the Fourier transform of tissue displacements during transient wave propagation (broad frequency band). The spectral information contained in this localized measurement, which is related to the inclusion viscoelasticity, should have the capability to allow the detection and characterization of very small mechanical heterogeneities. The finest resolution that can be reached is related to the calculated shear wave displacement map resolution, which is given by the axial and lateral sizes of one pixel of this map. In this report, each pixel of the wave displacement maps presented axial and lateral sizes equal to 0.288 and $0.296 \mathrm{~mm}$, respectively, and an axial averaging equal to $5.77 \mathrm{~mm}$.

As demonstrated in Figs. 4, 5, 7, 8, and 11, the first resonance-mode images depicted clear inclusion delineation compared to displacements in the surrounding material. This simple geometrical information that could be segmented visually may help to assess clot aging [39]-[41] or could complement other proposed techniques for DVT detection [42], [43]. More importantly, however, the quantitative assessment of blood clot elasticity proposed in the current study may further impact this field of research through the development of a new clinical imaging tool for patient management.

\section{B. Forward and Inverse Problem Validation}

The in vitro study, aiming to compare the assessed elasticity moduli with reference values for a large number of phantoms, permitted to quantitatively validate the DVT-SWIRE technique. In the forward problem, both experimental resonance frequency and 2-D displacement maps correlated well with simulated data, even for the challenging cases of a partially occluded lumen (Figs. 7 and 8). However, we noticed some differences close to the shearing source between 2-D stationary displacement fields measured for the total and partial clots in the case of the cylindrical wavefront excitation (Figs. 5 and 8). This can be attributed to potential slippage between the needle and the agar-gelatin material in experiments (the needle was simulated as firmly attached to the surrounding medium in the FEM model). This difference can also be due to the reflection of waves on the inclusion surface that was not perfectly vertical as implemented in the simulation.

The proposed approach formulated in the inverse problem successfully estimated the quantitative elasticity of mimicking thrombi with an excellent correlation with the reference measurements $\left(r^{2} \geq 0.986\right.$, Fig. 9). Nevertheless, as seen in Fig. 9(a) and Table III, the stiffer inclusions presented a higher deviation compared to the reference method. The main physical phenomenon underlying the SWIRE technique is the heterogeneity resonance that is possible when the surrounding tissue is stiffer than the confined inclusion. Consequently, the first resonance frequency is thus much more detectable in the inclusion spectrum when the contrast of elasticity is higher. For the phantoms $\# 8$ and $\# 9$, the stiffness contrast was reduced to 5.2, compared to 8.5 and 42.7 for the medium soft and very soft inclusions referred in Table I, respectively. Another cause of differences from perfect agreement with reference measurements could be attributable to temperature variations. As mentioned by Hall et al. [44], the agar-gelatin material is very sensitive to variations in temperature, and differences of even a few degrees Celsius could induce differences between the imaging and mechanical tests. 


\section{Ex Vivo Characterization of Fresh Animal Thrombi}

In this study, DVT-SWIRE was used to characterize mechanical parameters of fresh ex vivo pig thrombi one day after being surgically induced. Since the characterized vessels (vena cava and common iliac vein) are close to each other (Fig. 10), we expected that the thrombus formed along the diseased segments would have a homogeneous elasticity. This assumption was confirmed, as reported in Fig. 12.

To our knowledge, the only in vivo DVT stiffness evaluation, using quasi-static elastography and comparison with ex vivo mechanical tests, was proposed by Xie et al. [10] using a rat model of stasis-induced venous thrombosis. The results revealed an increase of thrombus elasticity as a function of time $(\mu=2.84 \pm$ $0.88 \mathrm{kPa}$ at day three postsurgery, $\mu=5.78 \pm 1.54 \mathrm{kPa}$ at day 6 , and $\mu=11.57 \pm 2.75 \mathrm{kPa}$ at day 10 ). They did not report the elasticity of thrombi $24 \mathrm{~h}$ postsurgery but their results showed that the thrombus elasticity doubled every day when perfused in vivo with circulating blood. By extrapolation, one can roughly approximate the elasticity for one-day old thrombi at around $710 \pm 220 \mathrm{~Pa}$, which is similar to the results of Fig. 12 . Nevertheless, it is important to modulate this quantitative comparison because major differences between their protocol and ours can be listed: rat versus pig model, quasi-static versus dynamic mechanical excitation at $85 \mathrm{~Hz}$, different blood hematocrits, no drug injection versus pro-coagulant (thrombin) injection, in vivo circulating blood perfusing condition versus same condition acutely for some hours followed by excision and measurements the day after ex vivo.

\section{Potential Limitations for Clinical Validation}

The current implementation of DVT-SWIRE relies on an experimental device requiring external vibrations to produce shear-horizontal plane or cylindrical shear waves. By sweeping the center frequency of a wavelet of a few cycles over a given bandwidth to induce resonance of the probed inclusion, its elasticity could be quantified with a FEM inverse problem strategy. Although the feasibility of DVT-SWIRE was proven for the experimental conditions defined in this study, this does not constitute a limitation since other configurations or parameter selection may also provide comparable results. It was not the aim of this study to evaluate the sensitivity of SWIRE to parameters such as the vibration amplitude, selected bandwidth, viscoelasticity contrasts or signal-to-noise ratios. Nevertheless, it is relevant to discuss some parameters of the current implementation of SWIRE with respect to known characteristics of ultrasound dynamic elastography methods.

In the current study, the external radiating pressure induced a tissue motion measured at the center of the inclusion, at resonance, of $\approx 100 \mu \mathrm{m}$ (see Fig. 6(a) for the cylindrical wavefront, similar displacements were measured in plane wavefront). As shown earlier [25], up to a ten time amplification of displacements at resonance is feasible compared to a nonresonant condition. Accordingly, a shear wave tissue vibration of $\approx 10 \mu \mathrm{m}$ within the DVT would be sufficient to produce SWIRE images since tissue motion estimators based on correlation techniques can have a tracking resolution as small as $1 \mu \mathrm{m}$ [45]. By using the supersonic shear wave imaging technique, a peak displacement of $10.6 \mu \mathrm{m}$ at a distance of $6.46 \mathrm{~mm}$ to the wave origin was noted [22], whereas a peak displacement of $25 \mu \mathrm{m}$ very close to the wave source was measured with the ARFI technique [46]. Those displacements induced by a localized vibrating source are compatible with SWIRE. Note that the vibrating sources of Fig. 2 were aligned with the mimicked blood clot for this proof-of-concept. It remains to be proven that resonance can be generated by using a radiation force polarization axis angulated with respect to the long axis of the blood vessel. With proper selection of cross-sectional or longitudinal view of the vessel and a quasi-plane shear wavefront angulated in the insonification plane, it may prove to be feasible. The impact of the detection angle between propagating shear waves and the ultrasound beam on the accuracy of speckle displacement detection remains to be evaluated.

Due to the inertia of the external vibrating source used in the current study, the wavelet excitation consisted of six oscillations modulated with a Blackman temporal window. To identify the first eigenmode of resonance, the center frequency of the shear wave excitation had to be swept from 45 to $205 \mathrm{~Hz}$ for the application in hand. In previous studies employing radiation pressure in supersonic imaging mode, one pulse of $2.55 \mathrm{~ms}$ was implemented [22]. This should be advantageous for SWIRE because of the wider bandwidth of this method. Indeed, one pulse excitation may be sufficient to cover the bandwidth of interest. In the current study, first resonance frequencies ranged from $57 \mathrm{~Hz}$ (for the very soft inclusion) to $156 \mathrm{~Hz}$ (for the stiffer one), which is equivalent to previous frequency bands measured in supersonic imaging mode [47].

As already noticed, the contrast of elasticity between the heterogeneity and the surrounding material has to be relatively high to ensure accurate mechanical parameter estimation. In the context of DVT, and in particular for the detection of recurrent thrombi, this condition should be true since the leg muscle shear elasticity is ranging from 9.9 to $24.9 \mathrm{kPa}$ [48], whereas fresh clots present few tens or hundreds of $\mathrm{Pa}$ [23], [24], [49]. This issue should thus not be considered as a potential limitation. Previous studies on the impact of muscle anisotropy (i.e., measurements along or across the muscle fibers) reported viscoelasticity variations up to $46 \%$ [50]-[52]. According to Fig. 14, varying the viscoelasticity of the surrounding medium (simulated muscle) by $40 \%$ still allowed robust estimates of the thrombus elasticity. Muscle anisotropy might thus not impact the SWIRE method.

\section{E. Towards an In Vivo Imaging Tool}

The results presented in this study showed that inclusion elasticity moduli evaluated using plane or cylindrical shear wavefronts (simulating a radiation pressure) are equivalent (Fig. 9). As already proposed by others [46], [53], a possible implementation of DVT-SWIRE for clinical applications should consider: 1) an ultrasound probe to image anatomical structures (i.e., a B-mode display), 2) a method to remotely generate shear waves at any desired positions by focusing ultrasound beams (to produce a radiation pressure locally at an optimal angle with respect to the DVT by using a proper electronic beamforming strategy), and 3) a strategy to track the 2-D wave displacement maps (in ultra-fast imaging mode) to recover the inclusion elasticity with the DVT-SWIRE inverse problem algorithm. DVT-SWIRE has 
the potential to be integrated into conventional scanners [46], [53], [54] without the need of new generation strategy to produce radiation pressure or new ultra-fast acquisition modalities. Indeed, the inclusion wave spectra can be obtained from 1-D data in Doppler [29], [55] or M-mode with a high frame rate, or using 2-D data acquired for a small region of interest [26], [46], [56]. All these possible configurations suggest potential real-time implementation using transient waves, and the integration of DVT-SWIRE in low cost conventional scanners or in a stand-alone dedicated imaging system. Of course finite element models can be time consuming if the geometry domain and mesh element dimensions are not suitable defined. Since the FEM was solved for each excitation frequency in order to determine the first resonance mode, the scanned range could be efficiently bounded by using a priori information such as the clot dimension and expected shear modulus. Another way to decrease the computation time of FEM would be to take advantage of parallel computing based on graphical processor units.

\section{CONCLUSION}

A novel imaging modality to characterize elastic properties of real and mimicking venous thrombi has been proposed and validated on realistic in vitro and animal ex vivo DVT phantoms. A simple but robust inverse problem was formulated to assess clot elasticity having different shapes (total or partial) and a wide range of elasticity moduli (from 406 to $3561 \mathrm{~Pa}$ ). The inverse problem strategy involved a 2-D FEM simulation that showed a good agreement with experimental data. Good correlations were found between reference measurements with the RheoSpectris instrument and those obtained with the DVT-SWIRE technique, for both wavefront configurations (plane and cylindrical) and clot geometries (lumen totally or partially occluded).

Further issues to explore would be to consider the viscosity evaluation using DVT-SWIRE since this mechanical parameter was proven to be modified during blood coagulation ([23], [24]). Since acoustic radiation force can remotely generate shear waves in tissues, SWIRE might therefore be combined with ARFI for in vivo thrombus aging experiments through measured viscoelastic properties. If implemented in a clinical scanner, the DVT-SWIRE may then be applied on patients and compared with conventional venous ultrasonography exams (i.e., compression ultrasonography, color Doppler).

\section{ACKNOWLEDGMENT}

The authors would like to thank Dr. H. Héon and N. Beauchemin for helping with the animal procedures. The authors gratefully acknowledge the support of Rheolution Inc. for the loan of the RheoSpectris instrument, and of the Institute of Biomedical Engineering of the ÉcolePolytechnique of Montreal for providing access to their computational FEM resources. The editorial assistance of A. Hollmann, Research Support Office, CHUM Research Centre, is also acknowledged.

\section{REFERENCES}

[1] R. H. White, "The epidemiology of venous thromboembolism," Circulation, vol. 107, no. 23, pp. I4-I8, Jun. 2003.

[2] P. A. Kyrle and S. Eichinger, "Deep vein thrombosis," Lancet, vol. 365, no. 9465 , pp. 1163-1174, Mar. 2005.
[3] C. Kearon, "Long-term management of patients after venous thromboembolism," Circulation, vol. 110, no. 9, pp. I10-I18, Aug. 2004.

[4] S. M. Bates and J. S. Ginsberg, "Clinical practice. Treatment of deepvein thrombosis," N. Eng. J. Med., vol. 351, no. 3, pp. 268-277, Jul. 2004.

[5] H. R. Buller, G. Agnelli, R. D. Hull, T. M. Hyers, M. H. Prins, and G. E. Raskob, "Antithrombotic therapy for venous thromboembolic disease: The seventh ACCP conference on antithrombotic and thrombolytic therapy," Chest, vol. 126, no. 3, pp. 401S-428S, Sep. 2004.

[6] W. J. Zwiebel, Introduction to Vascular Ultrasonography. New York: Elsevier, 2004, ch. 24.

[7] S. Y. Emelianov, X. Chen, M. O’Donnell, B. Knipp, D. Myers, T. W. Wakefield, and J. M. Rubin, "Triplex ultrasound: Elasticity imaging to age deep venous thrombosis," Ultrasound Med. Biol., vol. 28, no. 6 , pp. 757-767, Jun. 2002.

[8] B. Geier, L. Barbera, D. Muth-Werthmann, S. Siebers, H. Ermert, S. Philippou, and A. Mumme, "Ultrasound elastography for the age determination of venous thrombi. Evaluation in an animal model of venous thrombosis," Thromb. Haemost., vol. 93, no. 2, pp. 368-374, Feb. 2005

[9] H. Xie, K. Kim, S. R. Aglyamov, S. Y. Emelianov, X. Chen, M. O'Donnell, W. F. Weitzel, S. K. Wrobleski, D. D. Myers, T. W. Wakefield, and J. M. Rubin, "Staging deep venous thrombosis using ultrasound elasticity imaging: Animal model," Ultrasound Med. Biol., vol. 30, no. 10, pp. 1385-1396, Oct. 2004.

[10] H. Xie, K. Kim, S. R. Aglyamov, S. Y. Emelianov, M. O'Donnell, W. F. Weitzel, S. K. Wrobleski, D. D. Myers, T. W. Wakefield, and J. M. Rubin, "Correspondence of ultrasound elasticity imaging to direct mechanical measurement in aging DVT in rats," Ultrasound Med. Biol., vol. 31, no. 10, pp. 1351-1359, 2005.

[11] J. M. Rubin, H. Xie, K. Kim, W. F. Weitzel, S. Y. Emelianov, S. R. Aglyamov, T. W. Wakefield, A. G. Urquhart, and M. O'Donnell, "Sonographic elasticity imaging of acute and chronic deep venous thrombosis in humans," J. Ultrasound Med., vol. 25, no. 9, pp. 1179-1186, Sep. 2006.

[12] K. J. Parker, L. S. Taylor, S. Gracewski, and D. J. Rubens, "A unified view of imaging the elastic properties of tissue," J. Acoust. Soc. Am., vol. 117, no. 5, pp. 2705-2712, May 2005.

[13] A. P. Sarvazyan, O. V. Rudenko, S. D. Swanson, J. B. Fowlkes, and S. Y. Emelianov, "Shear wave elasticity imaging: A new ultrasonic technology of medical diagnostics," Ultrasound Med. Biol., vol. 24, no. 9 , pp. $1419-1435,1998$.

[14] L. S. Taylor, B. C. Porter, D. J. Rubens, and K. J. Parker, "Three-dimensional sonoelastography: Principles and practices," Phys. Med. Biol., vol. 45, no. 6, pp. 1477-1494, 2000.

[15] R. Muthupillai, D. J. Lomas, P. J. Rossman, J. F. Greenleaf, A. Manduca, and R. L. Ehman, "Magnetic-resonance elastography by direct visualization of propagating acoustic strain waves," Science, vol. 269, no. 5232, pp. 1854-1857, 1995.

[16] K. Daoudi, A.-C. Boccara, and E. Bossy, "Detection and discrimination of optical absorption and shear stiffness at depth in tissue-mimicking phantoms by transient optoelastography," Appl. Phys. Lett., vol. 94, no. 15, p. 154103, 2009.

[17] P. Asbach, D. Klatt, U. Hamhaber, J. Braun, R. Somasundaram, B. Hamm, and I. Sack, "Assessment of liver viscoelasticity using multifrequency MR elastography," Magn. Reson. Med., vol. 60, no. 2, pp. 373-379, Aug. 2008

[18] J. R. Basford, T. R. Jenkyn, K. N. An, R. L. Ehman, G. Heers, and K. R. Kaufman, "Evaluation of healthy and diseased muscle with magnetic resonance elastography," Arch. Phys. Med. Rehabil., vol. 83, no. 11, pp. 1530-1536, Nov. 2002.

[19] M. A. Green, L. E. Bilston, and R. Sinkus, "In vivo brain viscoelastic properties measured by magnetic resonance elastography," NMR Biomed., vol. 21, no. 7, pp. 755-764, Aug. 2008.

[20] L. Sandrin, B. Fourquet, J. M. Hasquenoph, S. Yon, C. Fournier, F. Mal, C. Christidis, M. Ziol, B. Poulet, F. Kazemi, M. Beaugrand, and R. Palau, "Transient elastography: A new noninvasive method for assessment of hepatic fibrosis," Ultrasound Med. Biol., vol. 29, no. 12, pp. 1705-1713, Dec. 2003.

[21] R. Sinkus, M. Tanter, T. Xydeas, S. Catheline, J. Bercoff, and M. Fink, "Viscoelastic shear properties of in vivo breast lesions measured by MR elastography," Magn. Reson. Imag., vol. 23, no. 2, pp. 159-165, Feb. 2005.

[22] M. Tanter, J. Bercoff, A. Athanasiou, T. Deffieux, J. L. Gennisson, G. Montaldo, M. Muller, A. Tardivon, and M. Fink, "Quantitative assessment of breast lesion viscoelasticity: Initial clinical results using supersonic shear imaging," Ultrasound Med. Biol., vol. 34, no. 9, pp. 1373-1386, Sep. 2008.

[23] J. L. Gennisson, S. Lerouge, and G. Cloutier, "Assessment by transient elastography of the viscoelastic properties of blood during clotting," Ultrasound Med. Biol., vol. 32, no. 10, pp. 1529-1537, Oct. 2006. 
[24] C. Schmitt, A. Hadj Henni, and G. Cloutier, "Characterization of blood clot viscoelasticity by dynamic ultrasound elastography and modeling of the rheological behavior," J. Biomech., vol. 44, no. 4, pp. 622-629, Feb. 2011.

[25] A. Hadj Henni, C. Schmitt, and G. Cloutier, "Shear wave induced resonance elastography of soft heterogeneous media," J. Biomech., vol. 43, no. 8, pp. 1488-1493, May 2010.

[26] A. Hadj Henni, C. Schmitt, and G. Cloutier, "Three-dimensional transient and harmonic shear-wave scattering by a soft cylinder for dynamic vascular elastography," J. Acoust. Soc. Am., vol. 124, no. 4, pp. 2394-2405, Oct. 2008.

[27] S. Catheline, J. L. Gennisson, G. Delon, M. Fink, R. Sinkus, S. Abouelkaram, and J. Culioli, "Measuring of viscoelastic properties of homogeneous soft solid using transient elastography: An inverse problem approach," J. Acoust. Soc. Am., vol. 116, no. 6, pp. 3734-3741, Dec. 2004.

[28] Q. C. Chan, G. Li, R. L. Ehman, R. C. Grimm, R. Li, and E. S. Yang, "Needle shear wave driver for magnetic resonance elastography," Magn. Reson. Med., vol. 55, no. 5, pp. 1175-1179, May 2006.

[29] M. Orescanin, K. S. Toohey, and M. F. Insana, "Material properties from acoustic radiation force step response," J. Acoust. Soc. Am., vol. 125, no. 5, pp. 2928-2936, May 2009.

[30] M. Yin, J. Woollard, X. Wang, V. E. Torres, P. C. Harris, C. J. Ward, K. J. Glaser, A. Manduca, and R. L. Ehman, "Quantitative assessment of hepatic fibrosis in an animal model with magnetic resonance elastography," Magn. Reson. Med., vol. 58, no. 2, pp. 346-353, Aug. 2007.

[31] J. Bercoff, M. Tanter, and M. Fink, "Supersonic shear imaging: A new technique for soft tissue elasticity mapping," IEEE Trans. Ultrason. Ferroelectr. Freq. Control, vol. 51, no. 4, pp. 396-409, Apr. 2004.

[32] A. Hadj Henni, C. Schmitt, M. É. Trembley, M. Hamdine, M.-C. Heuzey, P. Carreau, and G. Cloutier, "Hyper-frequency viscoelastic spectroscopy of biomaterials," J. Mechan. Behav. Biomed. Mater., vol. 4, pp. 1115-1122, 2011.

[33] D. G. Altman and J. M. Bland, "Measurement in medicine: The analysis of method comparison studies," Statistician, vol. 32, pp. 307-317, 1983.

[34] O. Lopez, K. K. Amrami, A. Manduca, and R. L. Ehman, "Characterization of the dynamic shear properties of hyaline cartilage using high-frequency dynamic MR elastography," Magn. Reson. Med., vol. 59, no. 2, pp. 356-364, Feb. 2008.

[35] K. R. Nightingale, M. L. Palmeri, R. W. Nightingale, and G. E. Trahey, "On the feasibility of remote palpation using acoustic radiation force," J. Acoust. Soc. Am., vol. 110, no. 1, pp. 625-634, Jul. 2001.

[36] W. F. Walker, F. J. Fernandez, and L. A. Negron, "A method of imaging viscoelastic parameters with acoustic radiation force," Phys. Med. Biol., vol. 45, no. 6, pp. 1437-1447, Jun. 2000.

[37] M. Fatemi and J. F. Greenleaf, "Ultrasound-stimulated vibro-acoustic spectrography," Science, vol. 280, no. 5360, pp. 82-85, Apr. 1998

[38] M. Fatemi and J. F. Greenleaf, "Vibro-acoustography: An imaging modality based on ultrasound-stimulated acoustic emission," in Proc. Nat. Acad. Sci., Jun. 1999, vol. 96, pp. 6603-6608.

[39] B. S. Hertzberg, M. A. Kliewer, D. M. DeLong, K. J. Lalouche, E. K. Paulson, M. G. Frederick, and B. A. Carroll, "Sonographic assessment of lower limb vein diameters: Implications for the diagnosis and characterization of deep venous thrombosis," AJR Am. J. Roentgenol., vol. 168, no. 5, pp. 1253-1257, May 1997.

[40] L. A. Linkins, P. Pasquale, S. Paterson, and C. Kearon, "Change in thrombus length on venous ultrasound and recurrent deep vein thrombosis," Arch. Int. Med., vol. 164, no. 16, pp. 1793-1796, Sep. 2004.
[41] P. Prandoni, A. Cogo, E. Bernardi, S. Villalta, P. Polistena, P. Simioni, F. Noventa, L. Benedetti, and A. Girolami, "A simple ultrasound approach for detection of recurrent proximal-vein thrombosis," Circulation, vol. 88, no. 4, pp. 1730-1735, Oct. 1993.

[42] J. Guerrero, S. E. Salcudean, J. A. McEwen, B. A. Masri, and S. Nicolaou, "System for deep venous thrombosis detection using objective compression measures," IEEE Trans. Biomed. Eng., vol. 53, no. 5, pp. 845-854, May 2006.

[43] J. Guerrero, S. E. Salcudean, J. A. McEwen, B. A. Masri, and S. Nicolaou, "Real-time vessel segmentation and tracking for ultrasound imaging applications," IEEE Trans. Med. Imag., vol. 26, no. 8, pp. 1079-1090, Aug. 2007.

[44] T. J. Hall, M. Bilgen, M. F. Insana, and T. A. Krouskop, "Phantom materials for elastography," IEEE Trans. Ultrason., Ferroelectr. Freq. Control, vol. 44, no. 6, pp. 1355-1365, Nov. 1997.

[45] D. Walker and G. E. Trahey, "A fundamental limit on the performance of correlation based on phase correction and flow estimation technique," IEEE Trans. Ultrason. Ferroelectr. Freq. Control, vol. 41, no. 5, pp. 644-654, 1994.

[46] M. L. Palmeri, M. H. Wang, J. J. Dahl, K. D. Frinkley, and K. R. Nightingale, "Quantifying hepatic shear modulus in vivo using acoustic radiation force," Ultrasound Med. Biol., vol. 34, no. 4, pp. 546-558, Apr. 2008

[47] T. Deffieux, G. Montaldo, M. Tanter, and M. Fink, "Shear wave spectroscopy for in vivo quantification of human soft tissues visco-elasticity," IEEE Trans. Med. Imag., vol. 28, no. 3, pp. 313-322, Mar. 2009.

[48] S. I. Ringleb, S. F. Bensamoun, Q. Chen, A. Manduca, K. N. An, and R. L. Ehman, "Applications of magnetic resonance elastography to healthy and pathologic skeletal muscle," J. Magn. Reson. Imag., vol. 25, no. 2, pp. 301-309, Feb. 2007.

[49] P. Riha, X. Wang, R. Liao, and J. F. Stoltz, "Elasticity and fracture strain of whole blood clots," Clin. Hemorheol. Microcirc., vol. 21, no. 1, pp. 45-49, 1999.

[50] M. W. Urban and J. F. Greenleaf, "A Kramers-Kronig-based quality factor for shear wave propagation in soft tissue," Phys. Med. Biol., vol. 54 , no. 19 , pp. 5919-5933, 2009.

[51] M. W. Urban, S. Chen, and J. F. Greenleaf, "Error in estimates of tissue material properties from shear wave dispersion ultrasound vibrometry," IEEE Trans. Ultrason. Ferroelectr. Freq. Control, vol. 56, no. 4, pp. 748-758, Apr. 2009.

[52] S. Chen, M. W. Urban, C. Pislaru, R. Kinnick, Y. Zheng, A. Yao, and J. F. Greenleaf, "Shearwave dispersion ultrasound vibrometry (SDUV) for measuring tissue elasticity and viscosity," IEEE Trans. Ultrason. Ferroelectr. Freq. Control, vol. 56, no. 1, pp. 55-62, Jan. 2009.

[53] J. Bercoff, A. Criton, C. C. Bacrie, J. Souquet, M. Tanter, J. L. Gennisson, T. Deffieux, M. Fink, V. Juhan, A. Colavolpe, D. Amy, and A. Athanasiou, "Shearwave elastography: A new real time imaging mode for assessing quantitatively soft tissue viscoelasticity," in IEEE Ultrason. Symp., 2008, pp. 321-324.

[54] S. McAleavey, E. Collins, J. Kelly, E. Elegbe, and M. Menon, "Validation of smurf estimation of shear modulus in hydrogels," Ultrason. Imag., vol. 31, no. 2, pp. 131-150, Apr. 2009.

[55] E. A. Barannik, A. Girnyk, V. Tovstiak, A. I. Marusenko, S. Y. Emelianov, and A. P. Sarvazyan, "Doppler ultrasound detection of shear waves remotely induced in tissue phantoms and tissue in vitro," Ultrasonics, vol. 40, no. 1-8, pp. 849-852, May 2002.

[56] H. Kanai, "Propagation of vibration caused by electrical excitation in the normal human heart," Ultrasound Med. Biol., vol. 35, no. 6, pp. 936-948, Jun. 2009. 